\title{
A Recent Development of Monitoring Devices on Smart Grid
}

\author{
Lilik. J. Awalin ${ }^{1}$, M. Khairil Rahmat ${ }^{2}$ \\ ${ }^{1}$ Universitas Airlangga, school of Advanced Technology and Multidisciplinary, Gedung Nanizar Zaman Joenoes, Kampus C Unair Jl. \\ Mulyorejo, Surabaya 60115, Indonesia. \\ ${ }^{2}$ Universiti Kuala Lumpur, British Malaysian Institute, Jalan Sungai Pusu Batu 8 Gombak 53100, Malaysia
}

\begin{abstract}
Reliability, safe and continuous electrical power supply are always bound to disruption and the urged of the advancement in technology has made developed countries to keep align with their current system especially in electrical power network. High demand in electrical technology has made researchers to find approach in inventing a smart meter measurement as to which technically has a big impact towards consumers. Considering the revolution of IoT sensing technology could aid the communication between the operator and the grid efficiently. Thus, the advancement of technology will boost the performance in wide area monitoring system.
\end{abstract}

Keywords: IoT sensing, Phasor Measurement Unit (PMU), Control Monitoring, Measurement, Smart Grid

\section{Introduction}

Conventional electrical energy management that has been done so far is only based on one Direction only, in the sense that electricity supply is only obtained from generators which are then forwarded to the transmission, distribution and proceed to the load network. By increasing of load, many problems have arisen regarding the supply of electrical energy. For that reason, in recent decades the Power and Energy System has relied on large solutions where Smart Grid will increase its efficiency and expenditure. In recent decades, the power and energy system are on the verge of a major change where Smart Grid solutions will increase their efficiency and flexibility. Enhanced information technology and control systems are key elements of the Smart Grid to enable efficient integration of large amounts of renewable energy resources which in turn seen as key elements of future energy systems.

Appropriate distribution grids must become more flexible and adaptable, for example in dealing with the high amount of energy that will come from renewable sources that are distributed. The complexity of Smart Grids needs to be considered and implies many components when a new application is designed.

Smart-Grid will also be able to control spending on electricity generated every day. That is because Smart Grid has four main components, namely generation, control, communication, and application. It will later be able to save electricity usage in homes. According to the paper [1], Smart grids can be defined as self-sufficient systems, which allows the integration of any type and any scale generation sources to the grid that reduces the workforce targeting sustainable, reliable, safe and quality electricity to all consumers.
To make a smart grid into a smart technology, several steps that must be done have been mentioned in the paper [2]. For the application of smart grids that are acceptable to the environment, some issues that must be resolved are also challenges for smart grid technology. Clean energy for environmental protection and scheduling of energy consumption patterns to meet its increasing demand is a challenge for the implementation of smart grids in the future [3].

On the other hand, as the smart technology in the electrical engineering field, standardization in the use of smart grids is very important as mentioned in the paper [4] - [5]. For example, in the US and Europe have tried to apply a standard in the application of smart grids. Although in the end, until now there are no definite provisions in the standard implementation of smart grid technology. Several approaches from various countries have also been developed to get a standard implementation of smart grid technology. Some countries have responded to this Smart-Grid technology quickly enough. Germany is one country with a very successful Smart-Grid model [6]. This paper states that Germany is a pioneer of implementing Smart-Grid through the City of Mannheim Model (MoMa) Project or the "Mannheim City Model" program. From the program, every home and office industry in the city of Mannheim will be integrated with an environmentally friendly electrical energy supply and anyone can see directly regulate the expenditure of electrical energy. This also makes the City of Mannheim as one of the smart cities or Smart-City which is quite popular. In 2014, this city was chosen as the best Smart-City city from The New Economy.

Adopting Germany's success in developing smart grid technology, there are several things that will be the 
focus of this paper, namely system monitoring which is one of the important components in the reliability of a smart grid system as discussed in paper [7]. Due to the importance of monitoring in smart grid applications, this paper will discuss developments in the last decade in the field of monitoring systems for smart grids. In this review, smart grid monitoring technology is divided into two parts, namely:

- Control monitoring of smart grids

- Measurement of smart grid

By having this review, researchers interested in this subject can benefit from the latest developments in smart grid technology. Therefore, accuracy in control and measurement systems can be done further. This paper is organized as follows; in the next section, part II, will present some of the research that has been done in the development of smart grid control technology. Part III deals with measurement components on smart grids. Then, Part IV will present the final conclusions.

\section{Control monitoring of smart grids}

In "Control of Flexible Smart Devices in the Smart Grid" paper, the author is concerned with investigating on the bidirectional communication link between the operator and the smart grid. In paper [8], a measurement with a flexible device is one of the important topics that the author highlighted. Especially on performing real time as accurately as possible for the data to be taken. In this paper, the author proposed on three algorithms where there are no control (NC) by means, if there is no communication or any commands that flows through between the controllers (supervisor) and flexible devices, then grid will not be flexible or nonflexible due to no permission has been instructed towards the device which then results in the grid will not act externally. Next is the threshold algorithm (TA) initiates control commands between the grid and the smart devices. The objective of this algorithm is to keep the load's system under a given capacity threshold regarding also its comfort and fairness issues. On/Off actions will be performed when the threshold is exceeded which almost similar with CCA algorithm. Meanwhile for continuous control algorithm (CCA) is a command to continuously exchange in and on/off schemes. The CCA command can be performed in two ways in which it can be performed within the smart grid or by the device level which resulting lower exchange of data between the agent (CPU) unit and the supervisors (controller). The principle of CCA is by sending commands to switch on/off to nonflexible into flexible loads at every time interval Tmin and the Tmax. Since CCA algorithm needs to be performed at different periods in a day making it as a preferable scheme. The whole idea of CCA algorithm is to minimize the consumption of energy. Paper [9] discuss the "Smart Power Meter to Monitor Energy Flow in Smart Grids: The Role of Advanced Sensing and IoT in the Electric Grid of the Future" is the potentialities of the sensing systems as well in controlling and monitoring of the smart grid with the aid of IoT. The revolution of the technology, making the current device needed improvements such that the presence of IoT could resolve challenges faced between the grid operator and the consumers. The author intends to present the advantages of having and IoT in the smart grid platform as to meet the optimization in power demand. Besides, the hardware and the software for the algorithm's functions were also being outlined by the author. The author also concerned more on the possibility in controlling and programming the meter to realize the remote mode and as for the remote-control station, it could be managed simultaneously on several smart meters.

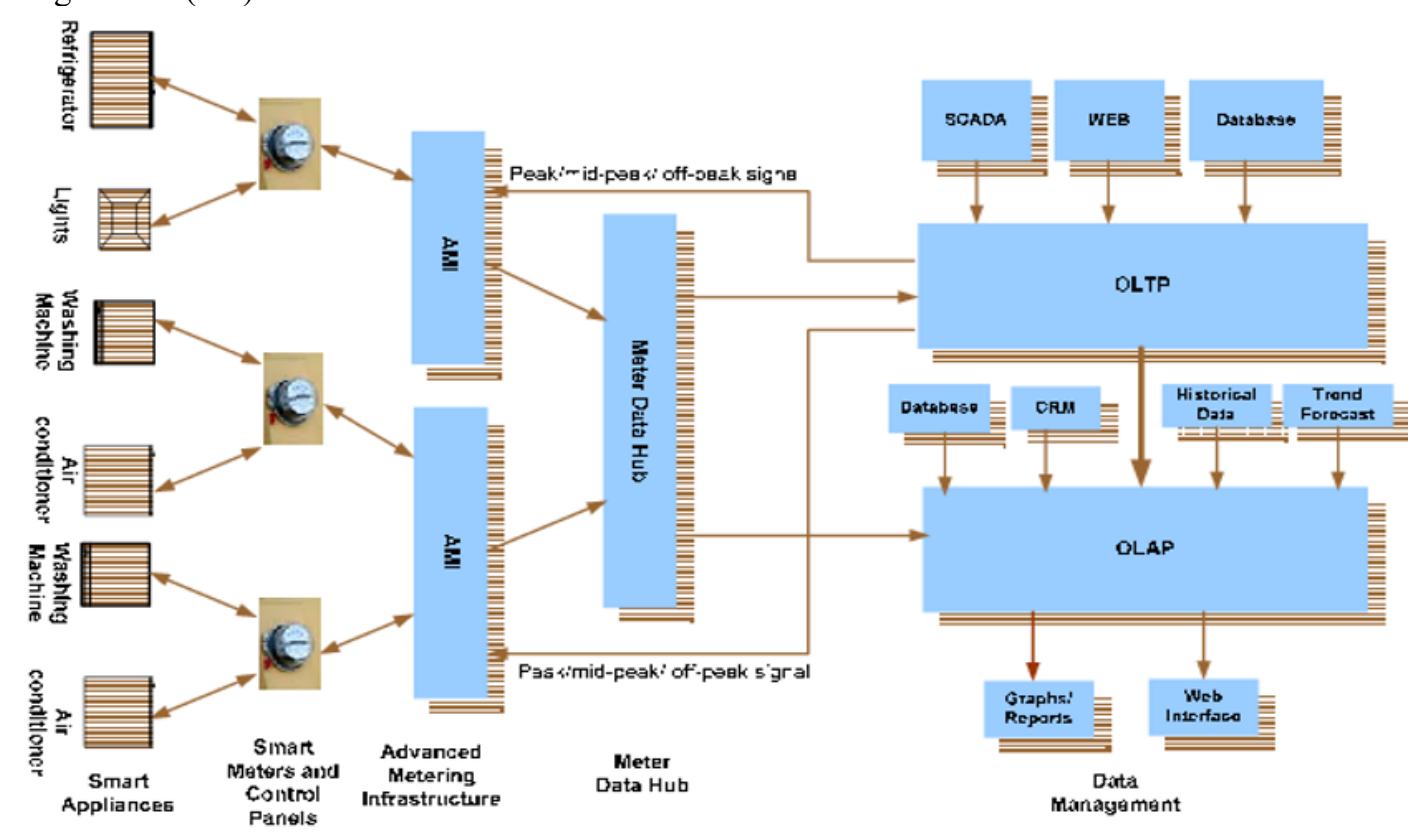

Fig. 1. Architecture of Real Time Smart Energy Management System with Smart Grid and Smart Appliances [10]. 


\section{Measurement of smart grid}

Measurement instrument is often related with scalar or vector measurement or even both. An instrument or a powerful tool is now developing each day with the revolution of technology that innovates the measurement instrument to become more sophisticated in terms of its infrastructures and functions. In electrical network, smart energy management is a vital key in running a reliable and safe system to ensure customer's satisfactions. Smart energy management involves in two major aspects which are the grid must be "smart" too, and the programmed of the smart meter.

On the other hand, paper [10] mention that the most important part in programming the smart meter is that it must possess the character of producing a real time and smart in controlling the grid itself. For instance, the panels are automatically controlled the grid to not only reduce the load variability.

In A Real-Time Architecture for Smart Energy Management paper presents the energy management system which constitutes of integral components such as the smart meter, the system used in modelling and processing the data and also the advance metering infrastructure as the central nervous system in transmitting and receiving the data which performs or react as a feedback loop. As to support the implementation of a smart meter, researchers keep on introducing and come up with algorithms or improving it as to solve the complexity of electrical network as so the smart meter can be realized within the smart grid. The highlight focus of the paper was, the architectural of the smart energy management is eased by the interfacing between online transactions processing (OLTP) and AMI. The structures of the OLTP consists of meter data, asset management, sales and billing, real time pricing, accounts payable and accounts receivable and portfolio management. The ability of the OLTP is that it performs dynamically signals where the supply information is obtained from SCADA centre, and OLTP will decides on whether the load is at either peak, mid-peak or on offpeak and the smart part is it relayed the signals with pricing information to the group customer examined using intelligent algorithm. The benefits of implementing the real time architecture are the power utility companies can control and the grid operations based on the demands, efficient trading the excess power with distribution side like pumping the water back to the reservoirs or changing the batteries and lastly could reduce the expenditure cost such as the maintenance of transformers, conductors and insulators when the historical data can be achieved significantly reliable. As the paced of the technology is acing day by day, many intelligence systems are combined to performed gianttech device such as the phasor measurement unit (PMU) used in electrical network. Based on Measurement Infrastructure to Support the Reliable Operation of Smart Electrical Grids paper. The author in paper [11] is concerned about the quality of the electricity supplied to the consumer due to multi resources of renewable energy are connected to the grid which results in a smart grid. In this paper, one of the key points in a measurement is that, the author is describing about the PMU device where it is one of the most powerful tools used in measuring electrical signals such as voltage, current and frequency. The author has mentioned that PMU device is implemented in a large electrical network or grid and likely be used in the future which is in medium and low voltage distribution grid. The framework design in the paper focused on the platform of the PMU where the simulation, the reference PMU and the metrology-grade calibrator are all connected towards each other in performing the function of the device. Through these combinations, PMU will be developed and evaluate the PMU algorithms, the calibrations and the results were to be compared with the field conditions. The author also breaks the PMU platform into its crucial components in complementing it such as the wave generator, interface of the algorithms and the error produced by the controlled of Graphical User Interface (GUI). The results of the signals produced by the wave generated was earlier discussed in paper [12]. As briefly explained in the paper, the phasor timetags constitute of a four (4) byte second-of-century (SOC) word in the Network Time Protocol (NTP) format and also two (2) byte sample count (SMPCNT) integer. Few observations can be taken into account based on the results observed which are; when using the timetag at the front window that results in the phasor to begin responding to the transient when the event ends. Besides, there are also an error that caused by the length of time the phasor needed to respond to the input. The author also explained that there are configurations file needed by the PMU which are a system configuration file CFG-1 and data configuration file CFG-2. Configurations include, station name and identification, number of phasors, number of digital channels, channel and phasor names, units, and conversion factors, nominal line frequency, transmission period (data rate).

Paper [13] also discuss a distribution System State Estimation Based on Non-synchronized Smart Meters. The author wanted to enhance the current system where the smart meter is not synchronized and the difference between the measurements in the smart meter is important. Thus, the author's highlighted parts on the important elements, such as the distribution network, smart grid and the smart meter itself. For the smart meter, the most top challenge is when the data taken is not synchronized or not taken in a real time. For instance, the electric variable is not taken on an exact time which results in the sampling time where these were caused by the lagging of the communication centre. Thus, the author is focusing on the distribution estimation based with the implementation meters where results proven by paper [14]. It was proved that the smart meter can be improved by the implementation of advance metering infrastructure where it can perform real time, measure, collecting and analyse the energy usage by providing real time load data. Power utility company can adopt load condition accurately by using state estimation on 16 bus systems and executed into AMI. Thus, making the author to investigate further on the impact of AMI data 
for typical state estimator. The simulation result is based on power solution values and some turbulences were added into the simulation of the AMI measurements. Based on Energy and Congestion-Aware Routing Metric for Smart Grid AMI Networks in Smart City paper, the author is focused on the network performance, which involved the functions of smart meter device by having an efficient routing metric to avoid energy constrained. The author adapting a parent node selection mechanism which considering the residual energy and utilizing the neighbourhood nodes. In this paper, the author has proven the scheme by using Cooja Simulator 3.0 by using random grid topology.

Paper [15] also discussed a dynamic parent node selection mechanism along with utilizing the queue. The author illustrates the connections of smart meters in RPL network connected with meter data management system (MDMS) via border router and IP network. Considering the advancement of the technology nowadays is widely deployed in many heavy industries, research paper of Advances on Sensing Technologies for Smart Cities and Power Grids: A Review, the author is towards the aim of describing the role of technologies such that the sensing systems able to deal with the challenges of a real time where the alerts are to be sent directly to mobile phones with the IoT based concept. Besides, the author also discussed the relationship between smart metering with the configuration of grid as there multi resources plant that supplied to the grid, making the energy flows in bidirectional. As a result, the amount of energy entering the nodes is unpredictable and consequence to this, the power quality becomes a matter as there are always disturbances in the signals. For supervision and control, paper [16] mention that it is very important to know the state of the grid by means the voltage and current phasors. To attend these, Phasor Measurement Units (PMU) have been developed to measure the parameters of the grid but the values are much higher. Thus, the usage of PMU is becoming more significant, especially in a wide area monitoring system (WAMS) as mentioned in paper [17]. The author's main aim is to differentiate between the two adapted techniques which is a frequency-adaptive approaches for PMU class $\mathrm{P}$ and $\mathrm{M}$ in order to achieve the wide frequency range. The first technique is a finite-impulse response (FIR) where there is no overshoot at the phase or the amplitude. The second technique is called Kalman filter-based (EKF) in which it allows the refinement of the out-of-band interference that has been rejected from the phase step response that has overshoot. In the FIR approach, it can generate a complex analytical signal $\mathrm{Xp}(\mathrm{t})$ corresponding to $\mathrm{x}(\mathrm{t})$ and then passing through the K-taps bandpass filter with respect to the filter center frequency. Meanwhile for Kalman filter, the algorithm designed as to be adapted by PMU relies on the fact that it can be performed such as in discrete Fourier transform with the aim to generate analytical signals at some of the given frequency components in the input side. The feature in which PMU is also important, is due to its capability in providing the dynamic performances [18] and algorithmic sophistication. The PMU also provides various performance levels such that Class-P is the faster
PMU and class-M is the slower PMU filter designs as discussed in paper [19]. In order to have a synchronize phasor, the author referred to [20] and [21] as to study the proposed algorithms that comply or achieved the similar requirements. Alongside of the study, the key performance index which is the Total Vector Error (TVE) is also being highlighted by the author such as the step and frequency response (gain and phase) characteristics covered under the amplitude and the phase modulation [22]. Based on WAMS Applications in Chinese Power Systems, the author also describing in details on the development of PMU device in monitoring the smart grid, which since 2002 to 2004, China has started to embark on the past technology which is the synchrophasor that has been developed for more than a decade. As one of the fastest developing country in the world. China is keeping its cities upgraded and equipped with the robust technologies where several manufacturers have come out with the PMU productions and Sifang is the first manufacturer that comply with the IEEE Standard 1344.

On the other hand, paper [23] present that the PMU device covers up on several crucial functions in controlling and monitoring the grid. For instance, the synchrophasor module adopts the signals from GPS and transferred it to other module to the equipment for synchronizing. There are three basic groups functions mentioned in the paper which categorized from B1 to B3 and B1 is where the platform form phasors' data, B2 is where the wide area is monitored and being analyzed and B3 is the synchronizing disturbance record and replay. Apart from the B functions, the author also described thoroughly on the advanced functions. The advance functions constitute from A1 until A9. There are basically, AF1 as the act as the generator operations status monitoring, AF2 as Online-Low-Frequency, and AF3 as Hybrid State Estimation. The author also stated some future developments that could be put into considerations such as more numbers of PMU will be deployed for instance plant size of 300MVA and above should be having the PMU device. Connecting the PMUs nationwide is also one of the possible ideas as almost every country is keeping up with deployment of Iot and sensing technology which could interconnecting the network and being conducted more efficiently when there are central stations as to control it. Meanwhile, in "Wide-Area Frequency Monitoring Network (FNET) Architecture and Applications" paper, the author is visualizing more on the architecture of FNET, where North America has used this since 2004 as to serve the power grid. FNET serves the grid by providing the situational awareness technique, real time functions, and accurate is estimation as part of the advantage is that the deployment of FNET is one of the lowest costs with high accuracy measurements as mention in paper [24]. These key elements played crucial role in controlling and monitoring of the grid to the most efficient level. The first synchrophasor prototype was built in 1988 at University (Virginia Tech) and up to this date as mentioned by the author, only 105 PMUs was installed. Alongside with the installation of the PMU, a frequency disturbance recorder (FDR) was also developed by the 
same University which was in 2003. There are about 20 of FDRs being installed as to combine with PMUs technology. The use of FDRs has been an easy as it is low in costs as each FDR itself is a single-phase PMU in which it measures the phase voltage, current and frequency. In "Synchronized Phasor Measurement Applications in Power Systems" paper, the author describing the crucial role played by the PMUs with the introduction of the device, the improvement made in its monitoring, control and protection in electrical networks. PMU has been introduced since early 1980s and booming towards the electrical industries as its features is being enhance day to day, time to time. For instance, past time uses synchrophasor method with the aid of algorithm and since then, the result has brought up to PMU which deployed in monitoring a wide area of the network along with phasor data concentrator (PDC) in hierarchical structure. The advantages of the PMU were explained on paper [25]. The data provide is accurate and is very important for the system to analysts and determine the sequence in which could analyze the causes of the breakdown of the power systems. One of the features in PMU is using the frequency-tracking step and estimating the period of the fundamental frequency before the phasor is being estimated. Furthermore, the technique used in phasor representation is a Fourier transform (DFT) which samples the data taken from input signals.

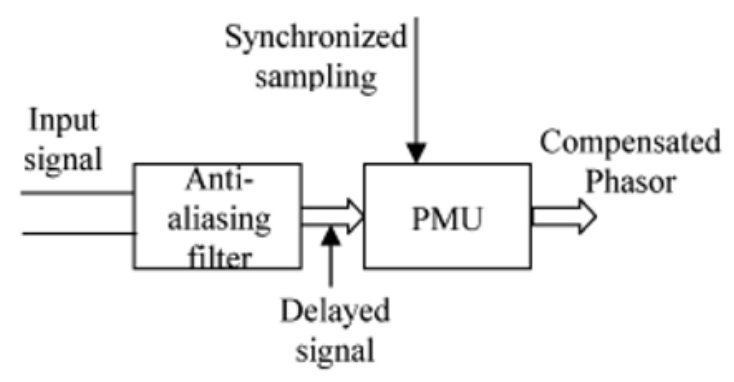

Fig. 2. Compensating for signal delay introduced by the antialiasing filter. [25].

Next, antialiasing filters are used as to limit the bandwidth was presented in paper [26]. It was supported by another researcher such as described in "Wide-Area Monitoring and Control Algorithms for Large Power Systems using Synchrophasors" paper, the constant challenges faced by the systems due to faults caused by the equipment and external faults. An overview of recent work done under Washington State University is on a real time monitoring implementation as in North America, the number of PMUs installation is increasing that urge the need to enhance the PMUs system so it can adapt on the dynamic monitoring as well. This outage of the systems can result in high stress towards the system and developed country such as Washington State keep on urging towards the better of reliable system by developing a real time monitoring and control algorithms as to cater the problems in the wide area monitoring system. Paper [26] also enlightens the vital tools that have been developed as the driving mechanism in order to have a fast detection and mitigation on any instability. The first tool introduced in the paper is a voltage stability monitoring by using data taken from PMU and by estimating the QV line sensitivity that connected to the bus and taking the sum of it as what recent papers such as mentioned in paper [27]. The author describing how the PMU could detect the voltage instability triggered by either transmissions or distributions and the results are to be told in a real time data by assuming the observability of the whole network that is prone to any of the voltage instability. The post-disturbance bus voltage is used as to monitor the outage. Any changes in the signs will be noticed through the set of equilibrium equations which adapted by the measurement device. The author also used a 52-bus 20-machine system as to simulate the feasibility of the PMU. Results were being illustrated by the author as to present the long-term voltage stability against time tested on the 52-bus system. The test system is also known as Nordic-32 test system which has been slightly modified. Some of the important changes notified in the results is at time $0.95 \mathrm{~s}$ where a three-phase short circuit is applied to the bus near 4044 and cleared after the $100 \mathrm{~ms}$ by isolating or open the faulted line 4032-4044. When the fault occurred, the system became insecure such that the reactive power is low due to its reservation towards some other generators and due to large power transfer from the North region to the Central areas. Thereby, the disturbances caused the system long-term voltage to be unstable. Consequence to that, unstable of the voltage will results in the loss of short-term stability which is produced in the form of field current and limited the generators due to the loss of its synchronism. Moreover, the effect of the noise could also give a negative impact on the PMUs performance as it could affect the sensitivities and leads to researchers to include the moving average (MA) filter on the input voltage side. For instance, the results obtained from the experiment is at $\mathrm{t}=45.7 \mathrm{~s}$, the sensitivities change sign for 60 seconds before the system collapses. Few works underway discussed in the paper is to have a quick identification on most efficient emergency controls, using more refined filters rather than MA, and test on faster restoring loads such as on induction motors. Next is the oscillation monitoring system (OMS) as to monitor the damping ratio, frequency and the mode shape [28]. The prototype of OMS has been developed and implemented in the Phasor Data Concentrator at TVA [29][26]. Finally, is using transient stability analysis by proposing algorithms that proposed by research paper [30] which is the mechanism for detection of the transient stability from the wide area monitored by measuring the bus voltage and its frequency. As said in "Optimal Design of a Wide Area Measurement System for Improvement of Power Network Monitoring Using a Dynamic Multiobjective Shortest Path Algorithm" paper described that there are three important elements in wide area monitoring system which are the management, measurement, and communication. The author in a paper [31] highlighted on the measurement and communication from the management viewpoint. First, the integer linear programming (ILP) is used for the optimal placement of the device by considering zero-injection bus effects. 
Next, the author also presenting the multi-objective shortest path programming as the aid in optimal placement as well. In terms of its architecture, an optical fiber power ground (OPGW) is the best architectural said by the author as the coverage for the suggested central control bus (CCB) and the number of PMUs. Paper [32] state that the PMU device also highly capable in synchronizing the measurement taken from other devices which possess the feature of higher precision and has a good real time monitoring for the system. In order to achieve the optimal result for the smart grid, the placement of the PMU is thus as crucial as its functions by taking all the consideration into account such as the effect of zero-injection buses as the key design in paper [31]. Lastly, from "Co-Optimal Placement of Measurement Devices and Their Related Communication Infrastructure in Wide Area Measurement Systems" research paper, the author mentioned the general elements under WAMS are data acquisition, data transmitting and data processing which carried out by the measurement device through communication systems. Thus, the placement of the measurement meter also played an important role in order to achieve the targeted goals and the infrastructure of communication weight the same important as the placement [33]. The author in a paper [33] describing the genetic algorithm (GA) problem as the approached in coping issues related to the monitoring. PMUs received data from global position system (GPS) and these data is used for synchronizing to have a common time reference also discussed in [34]- [35]. As to find the optimal placement of PMUs, the author in paper [34] simulates on few IEEE bus systems such as 14, 30,57 and 188-bus systems. The reason behind the placement of the PMU located at the busbar is it can measure the voltage phasor as well as the other end of the bus which is known as line parameters include the current phasor too. Researchers keep hunting the best method as to cater problems occurred in electrical network. For instance, the uncertainty of the voltage and phase angle along the transmission line were to be computed by the classical propagation theory. All results taken were tabulated as to see which parameter of it meets the requirement of the most optimal placement of the PMU. The experimental was carried out on an Intel Xeon 3.4-GHz CPU with a 2GB RAM. In summary, the integer quadratic programming approach in this paper could help to find the optimize location of the PMU.

\section{Conclusion}

This paper has presented an overview of measurement device used controlling and monitoring the smart grid. Every development found by the researchers gave big impact, especially on the features of the device such as the synchrophasor has been introduced since decade and has now turned into a robust and sturdy device which is Phasor Management Unit (PMU). The PMU is basically being classified into categories where there are class $\mathrm{P}$ and M PMU. The capability of this device makes so much difference in terms of the real time management where real time data is one of the key elements in adopting a wide area monitoring system (WAMS). This exercise also reveals research areas that can be conducted in the future to improve the existing device. Hence, such improvement could be one of the future works for improving the smart grid technology.

\section{Acknowledgements}

This work was supported by School of Advanced Technology and Multidisciplinary, Electrical Engineering Department, Airlangga University, Indonesia and FRGS Research Grant (Grant Code: FRGS/1/2018/TK04/UNIKL/02/3).

\section{References}

1. I. Colak, "Introduction to smart grid," 2016 International Smart Grid Workshop and Certificate Program (ISGWCP), Istanbul, 2016, pp. 1-5.

2. Collier, Steven E. "Ten steps to a smarter grid." IEEE Industry Applications Magazine 16, no. 2 (2010): 62-68.

3. Vineetha, C. P., and C. A. Babu. "Smart grid challenges, issues and solutions." In 2014 International Conference on Intelligent Green Building and Smart Grid (IGBSG), pp. 1-4. IEEE, 2014.

4. Rohjans, Sebastian, Mathias Uslar, Robert Bleiker, José González, Michael Specht, Thomas Suding, and Tobias Weidelt. "Survey of smart grid standardization studies and recommendations." In 2010 First IEEE International Conference on Smart Grid Communications, pp. 583-588. IEEE, 2010.

5. Andrén, Filip, Matthias Stifter, and Thomas Strasser. "Towards a semantic driven framework for smart grid applications: Model-driven development using CIM, IEC 61850 and IEC 61499." InformatikSpektrum 36.1 (2013): 58-68.

6. Wiseman, John, Taegen Edwards, and Kate Luckins. "Pathways to a sustainable and resilient urban future." In Resilient Sustainable Cities, vol. 31, no. 43, pp. 31-43. ROUTLEDGE in association with GSE Research, 2014.

7. Hao-Tian Zhang and Loi-Lei Lai, "Monitoring system for smart grid," 2012 International Conference on Machine Learning and Cybernetics, Xian, 2012, pp. 1030-1037.

8. Koutitas, G. (2012). Control of flexible smart devices in the smart grid. IEEE Transactions on Smart Grid, 3(3), 1333-1343.

9. Morello, R., De Capua, C., Fulco, G., \& Mukhopadhyay, S. C. (2017). A smart power meter to monitor energy flow in smart grids: The role of advanced sensing and IoT in the electric grid of the future. IEEE Sensors Journal, 17(23), 7828-7837.

10. Nagesh, D. R., Krishna, J. V., \& Tulasiram, S. S. (2010, January). A real-time architecture for smart 
energy management. In 2010 Innovative Smart Grid Technologies (ISGT) (pp. 1-4). IEEE.

11. Rietveld, G., Braun, J. P., Martin, R., Wright, P., Heins, W., Ell, N., ... \& Zisky, N. (2015). Measurement infrastructure to support the reliable operation of smart electrical grids. IEEE Transactions on Instrumentation and Measurement, 64(6), 1355-1363. [3,9250]

12. Martin, K. E., Benmouyal, G., Adamiak, M. G., Begovic, M., Burnett, R. O., Carr, K. R., ... \& Michel, G. L. (1998). IEEE standard for synchrophasors for power systems. IEEE Transactions on Power Delivery, 13(1), 73-77.

13. Alimardani, A., Therrien, F., Atanackovic, D., Jatskevich, J., \& Vaahedi, E. (2015). Distribution system state estimation based on nonsynchronized smart meters. IEEE Transactions on Smart Grid, 6(6), 2919-2928.

14. Jia, Z., Chen, J., \& Liao, Y. (2013, April). State estimation in distribution system considering effects of AMI data. In 2013 Proceedings of IEEE Southeastcon (pp. 1-6). IEEE.

15. Ullah, R., Faheem, Y., \& Kim, B. S. (2017). Energy and congestion-aware routing metric for smart grid AMI networks in smart city. IEEE access, 5, 1379913810.

16. Morello, R., Mukhopadhyay, S. C., Liu, Z., Slomovitz, D., \& Samantaray, S. R. (2017). Advances on sensing technologies for smart cities and power grids: A review. IEEE Sensors Journal, 17(23), 7596-7610.

17. Kamwa, I., Samantaray, S. R., \& Joos, G. (2013). Optimal integration of disparate C37. 118 PMUs in wide-area PSS with electromagnetic transients. IEEE transactions on power systems, 28(4), 47604770.

18. F. Steinhauser and Y. Ping, "Testing the dynamic response of phasor measurement units," in Proc. Int. Conf. Electr. Eng., 2009, pp. 1-5. [Online]. Available: http://www.iceecon.org/papers/2009/pdf/2.02_I9CP0546_E.Pdf

19. Das, S., \& Sidhu, T. (2013). A simple synchrophasor estimation algorithm considering IEEE standard C37. 118.1-2011 and protection requirements. IEEE Transactions on Instrumentation and Measurement, 62(10), 2704-2715.

20. I. Kamwa, A. K. Pradhan, and G. Joos, "Adaptive phasor and frequency-tracking schemes for widearea protection and control," IEEE Trans. Power Del., vol. 26, no. 2, pp. 744-753, Apr. 2011.

21. R. K. Mai, L. Fu, Z. Y. Dong, B. Kirby, and Z. Q. Bo, "An adaptive dynamic phasor estimator considering DC offset for PMU applications," IEEE Trans. Power Del., vol. 26, no. 3, pp. 1744-1754.

22. I. Kamwa, S. R. Samantaray, and G. Joos, "Compliance analysis of PMU algorithms and devices for wide-area stabilizing control of large power systems," IEEE Trans. Power Syst., vol. 28, no. 2, pp. 1766-1778, May 2013

23. Xie, X., Xin, Y., Xiao, J., Wu, J., \& Han, Y. (2006). WAMS applications in Chinese power systems. IEEE Power and Energy Magazine, 4(1), 54-63.

24. Zhang, Y., Markham, P., Xia, T., Chen, L., Ye, Y., Wu, Z., ... \& Conners, R. W. (2010). Wide-area frequency monitoring network (FNET) architecture and applications. IEEE Transactions on smart grid, 1(2), 159-167.

25. De La Ree, J., Centeno, V., Thorp, J. S., \& Phadke, A. G. (2010). Synchronized phasor measurement applications in power systems. IEEE Transactions on smart grid, 1(1), 20-27.

26. Venkatasubramanian, V., Yue, X., Liu, G., Sherwood, M., \& Zhang, Q. (2009, March). Widearea monitoring and control algorithms for large power systems using synchrophasors. In 2009 IEEE/PES Power Systems Conference and Exposition (pp. 1-5). IEEE.

27. Glavic, M., \& Van Cutsem, T. (2008, July). Detecting with PMUs the onset of voltage instability caused by a large disturbance. In 2008 IEEE Power and Energy Society General Meeting-Conversion and Delivery of Electrical Energy in the 21st Century (pp. 1-8). IEEE.

28. Liu, G., Quintero, J., \& Venkatasubramanian, V. M. (2007, August). Oscillation monitoring system based on wide area synchrophasors in power systems. In 2007 iREP symposium-bulk power system dynamics and control-VII. Revitalizing Operational Reliability (pp. 1-13). IEEE.

29. Liu, X., Liu, G., Sherwood, M., \& Venkatasubramanian, V. (2010, August). Wide-area monitoring and control algorithms for large power systems using synchrophasors. In 2010 IREP Symposium Bulk Power System Dynamics and Control-VIII (IREP) (pp. 1-5). IEEE.

30. Sherwood, M., Hu, D., \& Venkatasubramanian, V. M. (2007, August). Real-time detection of angle instability using synchrophasors and action principle. In 2007 iREP Symposium-Bulk Power System Dynamics and Control-VII. Revitalizing Operational Reliability (pp. 1-11). IEEE.

31. Ghasemkhani, A., Monsef, H., Rahimi-Kian, A., \& Anvari-Moghaddam, A. (2015). Optimal design of a wide area measurement system for improvement of power network monitoring using a dynamic multiobjective shortest path algorithm. IEEE Systems Journal, 11(4), 2303-2314.

32. Phadke, A. G., \& Thorp, J. S. (2008). Synchronized phasor measurements and their applications (Vol. 1, p. 81). New York: Springer.

33. Shahraeini, M., Ghazizadeh, M. S., \& Javidi, M. H. (2012). Co-optimal placement of measurement devices and their related communication infrastructure in wide area measurement systems. IEEE Transactions on Smart Grid, 3(2), 684-691. 
34. Chakrabarti, S., \& Kyriakides, E. (2007, August). Optimal placement of phasor measurement units for state estimation. In Proc. IASTED Int. Conf. Power Energy Syst. EuroPES, Palma de Mallorca, Spain (pp. 1-6).
35. Nuqui, R. F. (2001). State estimation and voltage security monitoring using synchronized phasor measurements (Doctoral dissertation, Virginia Tech). 